\title{
Editorial for Special Section on Health Psychology
}

The articles in this section are a continuation of the special edition in Health Psychology published in the earlier parts $(1,2)$ of this volume. The articles in this group deal with psychological factors in the aetiology, persistence, and progression of symptoms and disease.

Enduring characteristics of the individual have often been implicated as factors in the aetiology of disease. But traditional approaches to personality have yielded little fruit. Kamen and Seligman, however, describe an attributional style which predicts disease and mortality using a novel methodology. Personality traits are ruled out as an aetiological factor in Pearce's study of chronic pain; she postulates that the individual's response to adversity is more predictive. Finally, Levy and Wise examine the evidence that psychosomatic factors may critically influence the disease mechanisms in cancer prognosis and that changing coping style may influence the course of disease.

Thus, each article contributes to a growing body of evidence that coping style, conceptualized in various ways, is predictive of disease mechanisms.

Marie Johnston

Senior Lecturer in Clinical Psychology

Theresa Marteau Lecturer in Health Psychology 